\title{
Comparison of repeated applications of aPDT with amoxicillin and metronidazole in the treatment of chronic periodontitis: A short-term study
}

\author{
Letícia Helena Theodoro ${ }^{\mathrm{a}, *}$, Alessandra Barreto Lopes ${ }^{\mathrm{a}}$, Marta Aparecida Alberton Nuernberg ${ }^{\mathrm{a}}$, \\ Marina Módolo Cláudio ${ }^{\mathrm{a}}$, Daniela Maria Janjacomo Miessi ${ }^{\mathrm{a}}$, Márcio Luiz Ferro Alves ${ }^{\mathrm{a}}$, \\ Cristiane Duque ${ }^{\mathrm{b}}$, Andrea Mombelli ${ }^{\mathrm{c}}$, Valdir Gouveia Garcia ${ }^{\mathrm{a}, \mathrm{d}}$ \\ ${ }^{a}$ Department of Surgery and Integrated Clinic, Division of Periodontology, São Paulo State University (Unesp), School of Dentistry, Araçatuba, SP, Brazil \\ b Departament of Dentistry Children's and Social, São Paulo State University (Unesp), School of Dentistry, Araçatuba, SP, Brazil

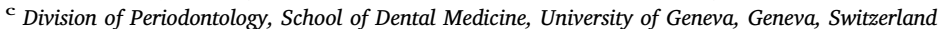 \\ d Latin American Institute of Dental Research and Education (ILAPEO), Curitiba, PR, Brazil
}

\section{A R T I C L E I N F O}

\section{Keywords:}

Periodontitis

Photochemotherapy

Dental Scaling

Antibiotics

\begin{abstract}
A B S T R A C T
Aim: The aim of this study was to compare the clinical effects of Metronidazole (MTZ) combined with Amoxicillin (AMX) and repeated applications of antimicrobial photodynamic therapy (aPDT) as an adjuvant for the treatment of chronic periodontitis.

Methods: A double-blind controlled and randomized clinical trial was conducted in 34 patients. All of the patients were treated with scaling and root planing (SRP) and separated into 2 groups: the MTZ + AMX Group ( $\mathrm{n}=17$ ), who received SRP and the systemic use of MTZ (400 mg $3 \times$ per day for 7 days) and AMX (500 mg $3 \times$ per day for 7 days), and the aPDT Group $(n=17)$, who received SRP and three aPDT applications at all sites with a probing depth $\geq 5 \mathrm{~mm}$ immediately, at 48 and $96 \mathrm{~h}$ after scaling and placebo pills over the span of 7 days. Clinical examinations were performed at baseline and 90 days post-therapy. The clinical parameters of bleeding on probing (BOP), probing depth (PD), and clinical attachment level (CAL) were measured. The aPDT was conducted using methylene blue and a low-level laser (GaAlAs $660 \mathrm{~nm}, 100 \mathrm{~mW}, 48 \mathrm{~s}$, and $160 \mathrm{~J} / \mathrm{cm}^{2}$ ) in all sites with a probing depth $\geq 5 \mathrm{~mm}$. A statistical analysis was also performed $(\alpha=5 \%)$.

Results: There was a significant improvement in CAL only in the intermediate pocket in the aPDT group compared to the MTZ + AMX group between baseline and 90 days post-treatment $(\mathrm{p}=0.01)$. There was a reduction of both BOP and the percentage of residual pockets at 90 days after treatment compared with baseline in both groups $(\mathrm{p}<0.05)$.

Conclusion: Both proposed adjuvant therapies associated with conventional mechanical treatment in patients with chronic periodontitis were equally effective in terms of the gain of clinical insertion, control of inflammation and elimination of residual pockets.
\end{abstract}

\section{Introduction}

Periodontitis is a chronic, multifactorial and polymicrobial inflammatory disease that leads to the destruction of tooth-supporting tissues $[1,2]$. The main purpose of periodontal therapy is to disorganize the bacterial biofilms and thereby reduce the number of periodontal microorganisms and control the disease [3]. Despite the positive clinical results obtained by using scaling and root planing (SRP) [4-7], which is the non-surgical periodontal therapy of choice, the effectiveness of alternative periodontal therapies has become essential to investigate as a consequence of the limited access to the root surface during the SRP [8-10] and due to the presence of periodontopathogens in the deeper periodontal pockets [11] and the ability of some bacteria to invade adjacent tissues [12].

Clinical studies have demonstrated that the systemic administration of amoxicillin (AMX) and metronidazole (MTZ), as adjuncts to nonsurgical mechanic periodontal treatment, are beneficial [2,13-31]. However, the effectiveness of antibiotic therapy in periodontics is still in question [32]. There are recommendations to restrict the use of antibiotics in periodontal therapy to limit the development of microbial antibiotic resistance. However, the true impact of the antibiotics used in periodontics on the resistance problem still needs to be elucidated [33-36].

In this context, antimicrobial photodynamic therapy (aPDT) has

\footnotetext{
* Corresponding author.

E-mail address: letheodoro@foa.unesp.br (L.H. Theodoro).
} 
been suggested as an alternative adjunctive antimicrobial procedure. It is characterized by the association of photosensitizing agents with different sources of light, such as lasers or light-emitting diodes (LED), with the goal of promoting the generation of species reactive to oxygen, such as free radicals and singlet oxygen, that are cytotoxic for some microorganisms [37]. In the action mechanism of aPDT, the photosensitizer absorbs the energy directly from the laser and transfers it to the oxygen molecules, thereby promoting the formation of reactive oxygen species (reaction type I) and singlet oxygen (type II reaction) $[38,39]$ that are highly cytotoxic to bacteria.

Clinical studies have demonstrated the effect of aPDT as an adjuvant therapy for the treatment of chronic periodontitis [40-48]. Some studies have shown a reduction in the percentage of bleeding on probing (BOP) in sites treated with aPDT $[42,44-46,49]$, whereas other studies have not shown clinical advantages with the use of aPDT $[45,47,50-52]$. Other clinical studies have shown a reduction of periodontopathogenic bacteria in sites treated with aPDT [46-48].

In addition, there are no reports in the literature on clinical comparisons of the use of multiple aPDT sessions with the use of systematic antibiotic therapy in chronic periodontitis. Given these facts, the aim of the present study was to clinically compare the effect of conventional periodontal therapy associated with either the combination of systemic MTZ and AMX or multiple applications of aPDT.

\section{Materials and Methods}

The present study was a randomized, prospective, controlled clinical trial with a 90-day follow up period. The study was approved by the Ethics and Human Research Committee of the Araçatuba Dental School, UNESP - CPE no. 173.653 e CAAE no. 05519412.5.0000.5420/2012.

\subsection{Calculation of the Sample}

The sample size was calculated regarding a difference of at least $1 \mathrm{~mm}$ in the pocket probing depth (PD) with a standard deviation of $0.90 \mathrm{~mm}$ between the groups in the deep pockets (PD $>6 \mathrm{~mm}$ ). It was determined that 13 patients per group would provide $80 \%$ power at a $5 \%$ level of significance (www.lee.dante.br).

\subsection{Selection of Patients and the Inclusion and Exclusion Criteria}

Ninety-eight patients from the Periodontal Clinic of the Araçatuba Dental School (UNESP) were evaluated. Thirty-four patients were included based on the following criteria: age $\geq 30$ years and $\leq 70$ years; diagnosed with severe generalized chronic periodontitis in at least 6 teeth and with one or several sites with $\mathrm{PD} \geq 5 \mathrm{~mm}$; a loss of clinical attachment level (CAL) $\geq 5 \mathrm{~mm}$; a minimum of $30 \%$ of the sites with $\mathrm{PD}$ and $\mathrm{CAL} \geq 4 \mathrm{~mm}$ and bleeding on probing (BOP); and the presence of at least 15 teeth when excluding the third molars. The exclusion criteria were as follows: periapical alterations in the qualified teeth; the need for antibiotic prophylaxis due to a systemic complication that influence the treatment response; periodontal treatment within the last 6 months; consumption of medicine that would affect the periodontal tissue within the last 6 months (antibiotics, anti-inflammatories, anticonvulsants, immunosuppressant or calcium channel blockers); allergies to penicillin or metronidazole; pregnant; in orthodontic treatment; presence of metabolic or immunological disorders; smokers or exsmokers; alcoholism; and the use of illicit drugs.

\subsection{Intra-examiner Calibration}

The calibration procedures for the treatments were conducted in 2 patients by a blind examiner. Therefore, two hundred seventy sites were analyzed in these two patients and PD in two different times (7-day interval). The data were submitted to the Kappa Concordance test, and the calibration was approved (0.89).

\subsection{Clinical Parameters}

All clinical exams were performed by a single examiner (ABL) and initially calibrated. Before starting treatment (baseline) and 90 days after treatment the examiner recorded the following parameters at six sites of every tooth: PD, BOP, and CAL. A millimeter periodontal probe was used for the clinical examination (PCPUNC-15, Hu-Friedy, Chicago, IL, USA).

The averages and standard deviations of the PD and CAL were calculated for each participant and for all of the sites in the mouth. The categorical BOP data were transformed into percentages, and the averages and standard deviations of the full mouth were obtained. The reduction of the number of residual pockets with $\mathrm{PD} \geq 5 \mathrm{~mm}$ and the percentage of sites with BOP were evaluated [31]. The individual risk profile for periodontal disease progression was evaluated according to Lang and Tonetti [53], as follows: low risk, no more than four sites with $\mathrm{PD} \geq 5 \mathrm{~mm}$; moderate risk, five to eight sites with $\mathrm{PD} \geq 5 \mathrm{~mm}$; and high risk, at least nine sites with $\geq 5 \mathrm{~mm}$ were evaluated.

\subsection{Treatment}

The 34 patients were randomly divided into two groups utilizing an online randomizer (www.sealedenvelope.com). Envelopes with the treatment distribution were prepared by a person independent of the clinical examiner (MLFA), and the envelopes were instructed to be opened only after SRP treatment. The participants were allocated to one of the following treatment groups: MTZ + AMX ( $n=17)$, which included SRP for one and a half hours, followed by the systemic administration of metronidazole $(400 \mathrm{mg})$ and amoxicillin $(500 \mathrm{mg}) 3$ times a day for 7 days [3], or aPDT ( $=17)$, which included SRP for one and a half hours, followed by the application of aPDT at all pockets $\geq 5 \mathrm{~mm}$ immediately, at 48 and $96 \mathrm{~h}$ after SRP and the administration of 2 placebo pills 3 times a day for 7 days.

The SRP and aPDT procedures were performed under local anesthesia by one operator (LHT) who specialized in periodontics. The placebo and antibiotics were administered immediately after the end of SRP.

aPDT was performed in all sites with a probing depth $\geq 5 \mathrm{~mm}$ in single- or multi-rooted teeth. The selected sites were irrigated with $1 \mathrm{ml}$ of $10 \mathrm{mg} / \mathrm{ml}$ of methylene blue (Aphoticário Pharmacy Manipulation, Araçatuba, SP, Brazil) utilizing a syringe and a blunt cannula (Becton Dickinson Ind. Ltd., Curitiba, PR, Brazil). After $1 \mathrm{~min}$, the sites were irradiated with a GaA1As diode laser at $660 \mathrm{~nm}$ (Laser Duo, MM Optics, Ltd., São Carlos, SP, Brazil) for $48 \mathrm{~s}$, which was equal to an energy density of $160 \mathrm{~J} / \mathrm{cm}^{2}$, an energy of $4.8 \mathrm{~J}$ with a power of $100 \mathrm{~mW}$, and fiber optics with a spot size of $0.03 \mathrm{~cm}^{2}$. The tip of the laser optic fiber was introduced to the area of the periodontal pocket, parallel to the long axis of the tooth, until it reached the bottom of the pocket. The patients and operator used specific protective glasses throughout the irradiation period.

\subsection{Compliance}

Patients were called 8 days after the beginning of the treatment for a clinical evaluation of the treatment. At this time, the medical history, use of concurrent medicine and any adverse effects were noted. Additionally, the patients were asked to bring their medicine bottles with them to determine the total use. The patients were also asked about the adverse effects of the medication.

\subsection{Statistical Analysis}

The data were submitted to statistical analysis using appropriate software (BioEstat 5.3 software, Mamirauá Institute, Manaus, Belém, PA Brazil). The clinical parameters were submitted to a normality test (Shapiro-Wilk test). Inter- and intragroup comparisons were performed 


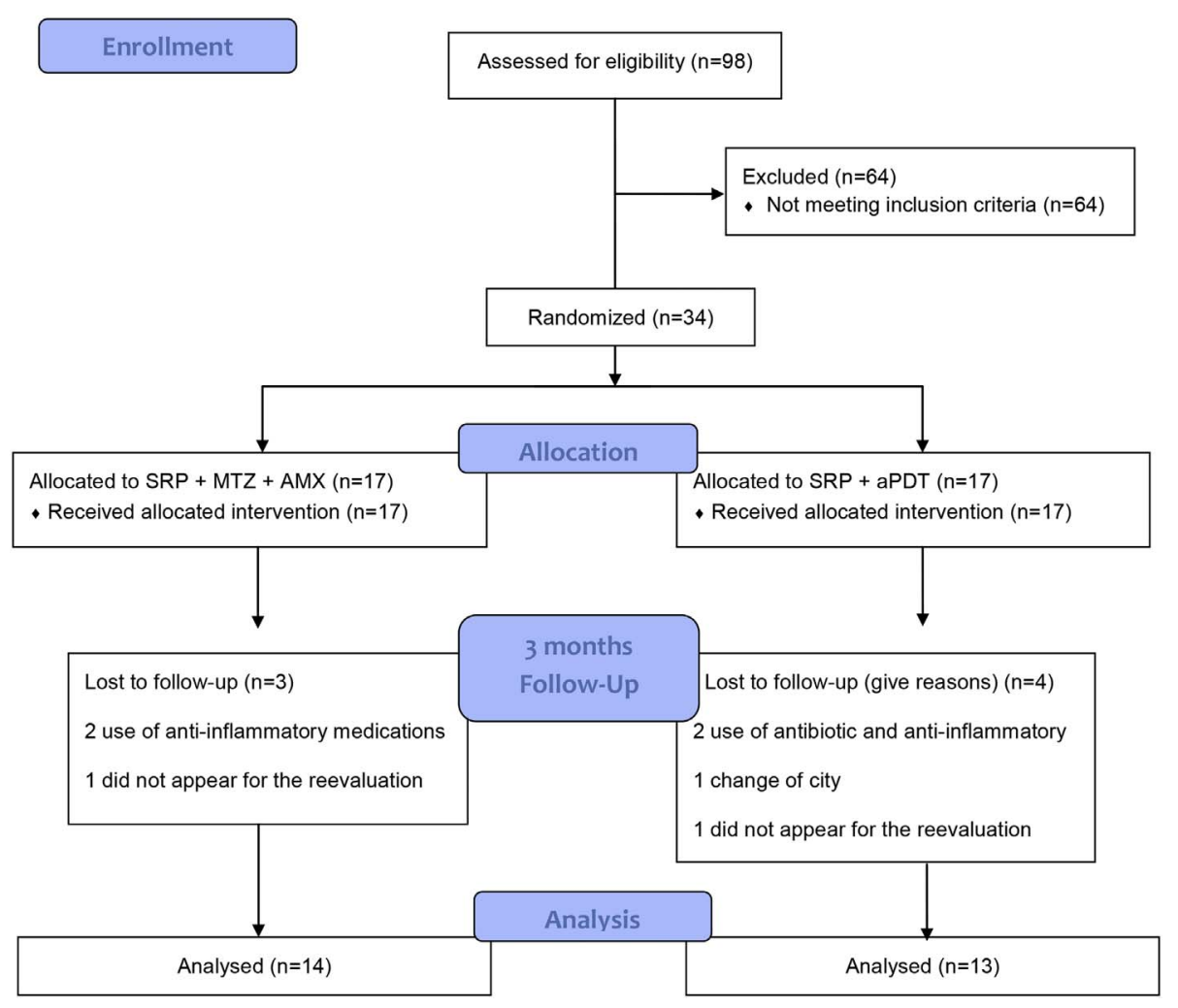

Fig. 1. Flowchart of study design.

for all of the variables at the different evaluation time points. Parametric data were submitted to a Student's $t$-test, and the nonparametric data were analyzed by the Wilcoxon test to compare the differences between the examination periods (baseline and 90 days) in each group. The Mann-Whitney test was performed to compare the differences between groups for non-parametric data, whereas parametric data were compared by an independent $t$-test. Chi-square test was used to compare the differences in the frequency of genders and compare subjects exhibiting different categories of individual risk profile for periodontal disease progression at 90 days of follow up. All statistical analyses considered a significance level of $5 \%$.

\section{Results}

All of the recruited patients (34) underwent the proposed treatments. Eighteen men and 16 women with an age of 32-66 years were treated. Of these 34 patients, 7 were excluded from the study due to the use of anti-inflammatory medications (2); not appearing for reevaluation (2); using antibiotic and anti-inflammatory medications (2); or a change of city (1) (Fig. 1).

Six patients from the MTZ + AMX group and one patient from the aPDT group reported gastrointestinal problems 8 days after the periodontal treatment.

\subsection{Clinical Findings}

The mean and standard deviation of the clinical parameters of the full mouth for the groups at each evaluation period are shown in Table 1. In the intergroup analysis, there was no differences in all of the clinical parameters values at baseline $(p>0.05)$. The intragroup analysis showed a significant improvement in all of the clinical parameters examined (BOP, PD and CAL) from baseline to 90 days posttreatment in both groups $(\mathrm{p}<0.05)$. In relation to the presence of residual pockets, there was a decrease in the number of residual pockets (PD $\geq 5 \mathrm{~mm}$ with BOP) in both treatments at 90 days (MTZ $+\mathrm{AMX}=13.57 \pm 11.67 \%$; $\mathrm{aPDT}=11.83 \pm 10.72 \%$ ) compared to
Table 1

Demographic characteristics and the mean and standard deviation $(M \pm S D)$ of the clinical parameters of the full mouth at baseline and 90-days after treatment.

\begin{tabular}{|c|c|c|c|}
\hline \multirow[t]{2}{*}{ Variable } & \multicolumn{2}{|l|}{ Groups } & \multirow[t]{2}{*}{ p-Value* } \\
\hline & $\begin{array}{l}\mathrm{MTZ}+\mathrm{AMX}^{\mathrm{a}} \\
\mathrm{n}=14\end{array}$ & $\begin{array}{l}\mathrm{aPDT}^{\mathrm{a}} \\
\mathrm{n}=13\end{array}$ & \\
\hline Age (years) & $46.3 \pm 6.8$ & $48.8 \pm 8.3$ & 0.38 \\
\hline Women, n (\%) & $7(50)$ & $5(38)$ & \\
\hline \multicolumn{4}{|l|}{$\mathrm{PD}(\mathrm{mm})$} \\
\hline Baseline & $3.99 \pm 0.57$ & $3.78 \pm 0.42$ & 0.42 \\
\hline 90 days & $3.69 \pm 0.38^{\mathrm{b}}$ & $3.54 \pm 0.41^{\mathrm{b}}$ & 0.25 \\
\hline \multicolumn{4}{|l|}{ CAL (mm) } \\
\hline Baseline & $4.91 \pm 1.20$ & $4.43 \pm 0.72$ & 0.22 \\
\hline 90 days & $4.62 \pm 1.07^{\mathrm{b}}$ & $4.25 \pm 0.71^{\mathrm{b}}$ & 0.33 \\
\hline \multicolumn{4}{|l|}{ BOP (\%) } \\
\hline Baseline & $75.04 \pm 19.09$ & $78.36 \pm 11.22$ & 0.58 \\
\hline 90 days & $60.58 \pm 15.95^{b}$ & $59.50 \pm 13.01^{b}$ & 0.84 \\
\hline \multicolumn{4}{|c|}{ Pockets with PD $\geq 5 \mathrm{~mm}$ (\%) } \\
\hline Baseline & $22.37 \pm 14.62$ & $17.98 \pm 10.43$ & 0.56 \\
\hline 90 days & $13.57 \pm 11.67^{\mathrm{b}}$ & $11.83 \pm 10.72^{\mathrm{b}}$ & 0.82 \\
\hline
\end{tabular}

${ }^{\mathrm{a}}$ Mean \pm standard deviation or number of participants (percentage).

b Statistically significant difference compared to the baseline of the same group.

* p-Value to MTZ + AMX vs aPDT.

the corresponding baseline data (MTZ + AMX $=22.37 \pm 14.62 \%$; $\mathrm{aPDT}=17.98 \pm 10.43 \%)(\mathrm{p}<0.01$; Table 1$)$.

In the analysis of intermediate pocket reduction, there was no difference between MTZ + AMX and aPDT groups for PD ( $p=0.30)$. However, there was a significant improvement in CAL in the intermediate pocket in the aPDT group compared to the MTZ + AMX group between baseline and 90 days post-treatment $(\mathrm{p}=0.01)$. Regarding the mean difference of the deep pocket reduction, there was no difference for PD and CAL between the treatment groups $(\mathrm{p}=0.49 ; \mathrm{p}=0.78)$ (Table 2).

All subjects included in this study presented high risk for disease progression (at least 9 sites with $\mathrm{PD} \geq 5 \mathrm{~mm}$ ) at baseline. After treatment four subjects (28.57\%) of MTZ + AMX group and five subjects 
Table 2

The mean difference and standard deviation $(M \pm S D)$ of the reduction of PD and CAL gain (intermediate and deep pockets) between the baseline and 90-days post-treatment.

\begin{tabular}{lll}
\hline Variable & & \\
\cline { 2 - 3 } & GTZ + AMX $^{\mathrm{a}}$ & p-Value $^{*}$ \\
$\mathrm{n}=14$ & $\mathrm{n}=13$
\end{tabular}

\begin{tabular}{|c|c|c|c|}
\hline \multicolumn{4}{|c|}{ Intermediate pockets } \\
\hline \multicolumn{4}{|c|}{$\mathrm{PD}(\mathrm{mm})$} \\
\hline 0-90 days & $0.53 \pm 0.23$ & $0.43 \pm 0.26$ & 0.30 \\
\hline \multicolumn{4}{|l|}{ CAL (mm) } \\
\hline 0-90 days & $0.46 \pm 0.43$ & $0.50 \pm 0.53$ & 0.01 \\
\hline \multicolumn{4}{|l|}{ Deep pockets } \\
\hline \multicolumn{4}{|l|}{ PD (mm) } \\
\hline $0-90$ days & $1.93 \pm 0.99$ & $1.64 \pm 1.08$ & 0.49 \\
\hline \multicolumn{4}{|l|}{ CAL (mm) } \\
\hline 0-90 days & $1.81 \pm 1.37$ & $1.65 \pm 1.16$ & 0.78 \\
\hline
\end{tabular}

a Mean difference \pm standard deviation

* p-Value to MTZ + AMX vs aPDT.

\section{Table 3}

Number and percentage of subjects presenting low, moderate and high risk for periodontal disease progression 90 days post-treatment. Mean \pm SD number of sites with shallow (PD $>3$ and $<5 \mathrm{~mm}$ ), intermediate ( $\mathrm{PD} \geq 5$ and $<7 \mathrm{~mm}$ ) and deep pockets (PD $\geq 7 \mathrm{~mm}$ ) at baseline and 90-days after treatment.

\begin{tabular}{llll}
\hline Variable & \multicolumn{2}{l}{ Groups } & p-Value \\
\cline { 2 - 3 } & $\begin{array}{l}\text { MTZ }+ \text { AMX } \\
\mathrm{n}=14\end{array}$ & $\begin{array}{l}\text { aPDT } \\
\mathrm{n}=13\end{array}$ & \\
\hline Low risk & - & - & \\
Moderate risk & $4(28.57 \%)$ & $5(38.46 \%)$ & $0.69^{\mathrm{a}}$ \\
High risk & $10(71.43 \%)$ & $8(61.54 \%)$ & \\
PD $>3$ and $<5 \mathrm{~mm}$ & & & \\
$\quad$ Baseline & $44.0 \pm 18.11$ & $34.64 \pm 14.99$ & $0.25^{*}$ \\
90 days & $41.50 \pm 20.96$ & $23.36 \pm 11.52$ & $0.70^{*}$ \\
PD $\geq 5$ and $<7 \mathrm{~mm}$ & & $18.21 \pm 9.43$ & $0.14^{*}$ \\
$\quad$ Baseline & $26.79 \pm 15.77$ & $11.43 \pm 8.71$ & $0.32^{*}$ \\
90 days & $18.50 \pm 15.57$ & & \\
PD $\geq 7$ mm & & $4.29 \pm 5.56^{\mathrm{b}}$ & $0.04^{*}$ \\
$\quad$ Baseline & $7.36 \pm 5.59$ & $3.0 \pm 5.07$ & $0.20^{*}$ \\
90 days & $4.71 \pm 3.83$ & & \\
\hline
\end{tabular}

${ }^{\text {a }}$ Chi-square test to compare subjects exhibiting different categories of individual risk profile for periodontal disease progression at 90 days post-treatment.

${ }^{\mathrm{b}}$ Statistically significant difference compared to MTZ + AMX group in the same point.

* p-Value to MTZ + AMX vs aPDT.

(38.46\%) of aPDT group achieved a moderate risk (5 to 8 sites with $\mathrm{PD} \geq 5 \mathrm{~mm}$ ) for future disease recurrence ( $\mathrm{p}=0.69$; Table 3 ). Table 3 presents the number of sites with shallow, intermediate and deep pockets at baseline and 90 days after treatment. Although not statistically significant, both groups exhibited a reduction in the number of these sites during the study.

\section{Discussion}

aPDT has been described as a secure procedure with potential improvements in clinical parameters when used in association with SRP [54]. In the present study, the treatment with multiple adjunctive applications of aPDT showed similar results to the effects of systemic antibiotics administered for the treatment of chronic periodontitis with a 90-day follow-up.

Studies in adults with chronic periodontitis have shown that the use of an antimicrobial agent as an adjunct to mechanical therapy is more effective in periodontal treatment than isolated SRP therapy [32,55]. According to a meta-analysis, the use of systemic antibiotics in the nonsurgical treatment of periodontitis provides a statistically significant reduction of PD $(0.55 \pm 0.37 \mathrm{~mm}$, average $)$ after MTZ + AMX use compared to isolated SRP 12 months post-treatment [55]. In the treatment of chronic periodontitis, a majority of studies have used the combination of MTZ + AMX for a period of 7 days $[15,16,18-20,30,31,56-64]$; however, some authors used it for a period of 14 days [17,25,35,65-67].

Despite the satisfactory clinical results, antibiotic therapy usually leads to undesirable side effects involving the digestive system and genitourinary tract as well as the development of bacterial resistance. To minimize such effects, the search for local antimicrobial methods for the treatment of periodontitis continues to be widely investigated [68-70].

Previous studies with aPDT as an adjuvant to SRP have shown greater PD reductions and significant clinical insertion gains in treated sites compared to isolated SRP in the initial periodontal therapy of patients with chronic periodontitis $[37,43]$. The beneficial microbiological effect of aPDT as an adjunct to conventional mechanical treatment of periodontal disease is probably a result of the photodestructive effects of oxygen-reactive species and by the modulation action of the low level laser [47]. The oxygen-reactive species lead an irreversible damage to the bacterial cytoplasmic membrane, including protein modification, respiratory chain breakdown and nucleic acid alterations [71].

The number of aPDT application sessions seems to be directly related to the success of the therapy. When aPDT was used in a single session, it did not verify the clinical improvements in PD and CAL for chronic periodontitis treatment $[47,50]$, although aPDT was able to reduce the BOP of the sites treated $[44,45,49]$. Nevertheless, the evaluation of multiple applications of aPDT effects on the periodontal treatment of residual pockets showed clinical improvements with a reduction of PD with reduced bleeding [72,73]. Another study that performed three applications of aPDT in residual pockets on the first, third and seventh days after the SRP did not show clinical improvements in relation to the PD and CAL parameters but found a greater BOP reduction at 3 and 12 months compared to isolated SRP [46].

In the present study, there was a significant reduction in the percentage of residual pockets in both groups at 90 days post-treatment. The resolution of residual pockets remains a challenge for conventional periodontal therapy because several SRP sessions may not increase the success of treatment in the periodontal pockets [74,75]. Furthermore, both treatments resulted in a decrease of the number of sites with $\mathrm{PD} \geq 5 \mathrm{~mm}$. Four patients (28.57\%) at MTZ + AMX group and five patients $(38.46 \%)$ at aPDT group achieved a moderate risk for future disease recurrence according to Lang and Tonetti at 90 days posttreatment. In this study, there was significant improvement in CAL in intermediate pocket in the aPDT group compared to the MTZ + AMX group between baseline and 90 days post-treatment. The present results suggest that the use of systemic antibiotics and three sessions of aPDT confer similar benefits as does a non-surgical periodontal treatment, which decreases the recurrence of periodontal disease in the short term and causes less damage to mineralized dental tissues.

Regarding BOP, there was a significant reduction in the MTZ + AMX $(p=0.0007)$ and aPDT $(p<0.0001)$ group in the 90-day period. These results were corroborated in another study that showed a decrease in the clinical signs of inflammation and inflammatory biomarkers in crevicular fluid with the association of MTZ and AMX with SRP [60]. Concerning aPDT, a previous study showed that sites treated with aPDT showed increased levels of IL-4 and IL-10 (anti-inflammatory) and a reduction of GM-CSF, IL-1b, and IL-6 (pro-inflammatory), which provided significant modulation in the cytokines level [76]. Thus, the proposed adjuvant therapies can decrease the chance of retreatment, lower the costs of repeated treatments, and avoid exposing the patient to several SRP sessions, which could also promote mineralized tissue damage and gingival recession.

Comparing the use of antibiotic therapy (375 mg of AMX $+250 \mathrm{mg}$ of MTZ) with two applications of aPDT in the aggressive periodontitis treatment, the use of the antibiotic therapy showed more benefits [77]. However, in the present study, the protocol with three aPDT 
applications with 48-h intervals proved to be as effective as antibiotic therapy in the chronic periodontitis treatment.

The conflicting results in the aPDT studies can be justified due to the different methodologies and protocols used in clinical studies. Several factors may influence the aPDT as a characteristic and concentration of the photosensitizer used, duration of pre-irradiation, irradiation time, light application devices, wavelengths and light output power [50,78]. Thus, these disagreements between studies may influence the clinical outcomes and prevent direct comparisons between the analysis [37].

Recently, a systematic review and meta-analysis on the nonsurgical treatment of patients with chronic periodontitis by means of SRP with or without adjuncts was conducted. The panel judged the following 4 adjunctive therapies as beneficial with a moderate level of certainty: systemic subantimicrobial-dose doxycycline, systemic antimicrobials, chlorhexidine chips, and photodynamic therapy with a diode laser [79]. Compared with SRP alone, SRP plus the aPDT resulted in a $0.53 \mathrm{~mm}$ mean gain in CAL (0.06-1.00), and SRP plus systemic antibiotic resulted in a $0.35(0.20-0.51)$ mean gain in CAL [79]. In the present study, aPDT resulted in a $0.50(0.53)$ mean gain in CAL, and systemic antibiotic resulted in $0.46(0.43)$ mean gain in CAL in the moderate pocket, whereas in the deep pockets, aPDT resulted in a 1.65 (1.16) mean gain in CAL and systemic antibiotic resulted in a 1.81 (1.37) mean gain in CAL.

In this study, all patients had severe generalized chronic periodontitis. Most patients in this study had never received adequate periodontal treatment or instruction on oral hygiene before being admitted in this public service at the university, probably because they had a low socioeconomic status. Future investigations should be conducted to establish clinical protocols for aPDT in chronic periodontitis. It is also suggested that more controlled and randomized clinical studies with complementary analyses should be conducted to verify the differences in antimicrobial and inflammatory behavior among the studied therapies. aPDT presents additional benefits because it is a local therapy and does not cause side effects or the resistance of bacterial strains.

Despite the limitations of the present study in a short term, it can be concluded that both of the proposed adjuvant therapies, which are associated with conventional mechanical treatment in patients with chronic periodontitis, were equally effective in the gain of clinical insertion, the control of inflammation and the elimination of residual pockets.

\section{Conflict of Interest Statement}

The authors have declared no conflict of interest.

\section{Acknowledgements}

This study was supported in part by FAPESP (Support Foundation of São Paulo Research, Process \# 2014/11637-2) and the CNPq (National Counsel of Technological and Scientific Development - Process \# 302806/2012-1).

\section{References}

[1] J. Cazalis, et al., Tetracyclines and chemically modified tetracycline-3 (CMT-3) modulate cytokine secretion by lipopolysaccharide-stimulated whole blood, Inflammation 32 (2) (2009) 130-137.

[2] D. Zandbergen, et al., The clinical effect of scaling and root planing and the concomitant administration of systemic amoxicillin and metronidazole: a systematic review, J. Periodontol. 84 (3) (2013) 332-351.

[3] S.S. Socransky, A.D. Haffajee, Dental biofilms: difficult therapeutic targets, Periodontology 28 (2000) 12-55 2002.

[4] A. Badersten, R. Nilveus, J. Egelberg, Effect of nonsurgical periodontal therapy. I. Moderately advanced periodontitis, J. Clin. Periodontol. 8 (1) (1981) 57-72.

[5] L.H. Carvalho, et al., Scaling and root planing, systemic metronidazole and professional plaque removal in the treatment of chronic periodontitis in a Brazilian population. I. Clinical results, J. Clin. Periodontol. 31 (12) (2004) 1070-1076.

[6] A.D. Haffajee, et al., The effect of SRP on the clinical and microbiological parameters of periodontal diseases, J. Clin. Periodontol. 24 (5) (1997) 324-334.
[7] C.M. Cobb, Clinical significance of non-surgical periodontal therapy: an evidencebased perspective of scaling and root planing, J. Clin. Periodontol. 29 (Suppl. 2) (2002) 6-16.

[8] P.A. Adriaens, J.A. De Boever, W.J. Loesche, Bacterial invasion in root cementum and radicular dentin of periodontally diseased teeth in humans. A reservoir of periodontopathic bacteria, J. Periodontol. 59 (4) (1988) 222-230.

[9] A. Aoki, et al., Lasers in nonsurgical periodontal therapy, Periodontology 36 (2000) 59-97 2004.

[10] A.A. Takasaki, et al., Application of antimicrobial photodynamic therapy in periodontal and peri-implant diseases, Periodontology 51 (2000) 109-140 2009.

[11] F.W. Muniz, et al., Azithromycin: a new concept in adjuvant treatment of periodontitis, Eur. J. Pharmacol. 705 (1-3) (2013) 135-139.

[12] A. Amano, Disruption of epithelial barrier and impairment of cellular function by Porphyromonas gingivalis, Front. Biosci. 12 (2007) 3965-3974.

[13] A.J. van Winkelhoff, et al., Metronidazole plus amoxycillin in the treatment of Actinobacillus actinomycetemcomitans associated periodontitis, J. Clin. Periodontol. 16 (2) (1989) 128-131.

[14] M.J. Pavicic, et al., Microbiological and clinical effects of metronidazole and amoxicillin in Actinobacillus actinomycetemcomitans-associated periodontitis. A 2year evaluation, J. Clin. Periodontol. 21 (2) (1994) 107-112.

[15] E.G. Winkel, et al., Amoxicillin plus metronidazole in the treatment of adult periodontitis patients. A double-blind placebo-controlled study, J. Clin. Periodontol. 28 (4) (2001) 296-305.

[16] J. Rooney, et al., Adjunctive effects to non-surgical periodontal therapy of systemic metronidazole and amoxycillin alone and combined. A placebo controlled study, J. Clin. Periodontol. 29 (4) (2002) 342-350.

[17] F. Matarazzo, et al., Clinical and microbiological benefits of systemic metronidazole and amoxicillin in the treatment of smokers with chronic periodontitis: a randomized placebo-controlled study, J. Clin. Periodontol. 35 (10) (2008) 885-896.

[18] N. Cionca, et al., Amoxicillin and metronidazole as an adjunct to full-mouth scaling and root planing of chronic periodontitis, J. Periodontol. 80 (3) (2009) 364-371.

[19] P. Ribeiro Edel, et al., Full-mouth ultrasonic debridement associated with amoxicillin and metronidazole in the treatment of severe chronic periodontitis, J. Periodontol. 80 (8) (2009) 1254-1264.

[20] N. Cionca, et al., Microbiologic testing and outcomes of full-mouth scaling and root planing with or without amoxicillin/metronidazole in chronic periodontitis, J. Periodontol. 81 (1) (2010) 15-23.

[21] M.J. Mestnik, et al., Short-term benefits of the adjunctive use of metronidazole plus amoxicillin in the microbial profile and in the clinical parameters of subjects with generalized aggressive periodontitis, J. Clin. Periodontol. 37 (4) (2010) 353-365.

[22] E.C. Yek, et al., Efficacy of amoxicillin and metronidazole combination for the management of generalized aggressive periodontitis, J. Periodontol. 81 (7) (2010) 964-974.

[23] G.S. Griffiths, et al., Amoxicillin and metronidazole as an adjunctive treatment in generalized aggressive periodontitis at initial therapy or re-treatment: a randomized controlled clinical trial, J. Clin. Periodontol. 38 (1) (2011) 43-49.

[24] D. Heller, et al., Impact of systemic antimicrobials combined with anti-infective mechanical debridement on the microbiota of generalized aggressive periodontitis: a 6-month RCT, J. Clin. Periodontol. 38 (4) (2011) 355-364.

[25] M.P. Silva, et al., Clinical and microbiological benefits of metronidazole alone or with amoxicillin as adjuncts in the treatment of chronic periodontitis: a randomized placebo-controlled clinical trial, J. Clin. Periodontol. 38 (9) (2011) 828-837.

[26] V.M. Varela, et al., Systemic antimicrobials adjunctive to a repeated mechanical and antiseptic therapy for aggressive periodontitis: a 6-month randomized controlled trial, J. Periodontol. 82 (8) (2011) 1121-1130.

[27] M. Feres, et al., Metronidazole alone or with amoxicillin as adjuncts to non-surgical treatment of chronic periodontitis: a 1-year double-blinded, placebo-controlled, randomized clinical trial, J. Clin. Periodontol. 39 (12) (2012) 1149-1158.

[28] M.J. Mestnik, et al., The effects of adjunctive metronidazole plus amoxicillin in the treatment of generalized aggressive periodontitis: a 1-year double-blinded, placebocontrolled, randomized clinical trial, J. Clin. Periodontol. 39 (10) (2012) 955-961.

[29] F. Sgolastra, et al., Effectiveness of systemic amoxicillin/metronidazole as adjunctive therapy to scaling and root planing in the treatment of chronic periodontitis: a systematic review and meta-analysis, J. Periodontol. 83 (10) (2012) 1257-1269.

[30] A. Mombelli, et al., Are there specific benefits of amoxicillin plus metronidazole in Aggregatibacter actinomycetemcomitans-associated periodontitis? Double-masked, randomized clinical trial of efficacy and safety, J. Periodontol. 84 (6) (2013) $715-724$.

[31] A. Mombelli, et al., Differential benefits of amoxicillin-metronidazole in different phases of periodontal therapy in a randomized controlled crossover clinical trial, J. Periodontol. 86 (3) (2015) 367-375.

[32] A. Mombelli, N. Cionca, A. Almaghlouth, Does adjunctive antimicrobial therapy reduce the perceived need for periodontal surgery, Periodontology 55 (1) (2000) 205-216 2011.

[33] A. Mombelli, et al., Effect of periodontal therapy with amoxicillin-metronidazole on pharyngeal carriage of penicillin- and erythromycin-resistant Viridans streptococci, J. Periodontol. 87 (5) (2016) 539-547.

[34] A.J. van Winkelhoff, et al., Antimicrobial resistance in the subgingival microflora in patients with adult periodontitis. A comparison between The Netherlands and Spain, J. Clin. Periodontol. 27 (2) (2000) 79-86.

[35] M. Feres, et al., Antibiotic resistance of subgingival species during and after antibiotic therapy, J. Clin. Periodontol. 29 (8) (2002) 724-735.

[36] C.M. Ardila, M.I. Granada, I.C. Guzman, Antibiotic resistance of subgingival species in chronic periodontitis patients, J. Periodontal Res. 45 (4) (2010) 557-563.

[37] M.G. Correa, et al., Short-term microbiological effects of photodynamic therapy in 
non-surgical periodontal treatment of residual pockets: a split-mouth RCT, Lasers Surg. Med. 48 (10) (2016) 944-950.

[38] M. Ochsner, Photophysical and photobiological processes in the photodynamic therapy of tumours, J. Photochem. Photobiol. B 39 (1) (1997) 1-18.

[39] J.P. Tardivo, et al., Methylene blue in photodynamic therapy: from basic mechanisms to clinical applications, Photodiagn. Photodyn. Ther. 2 (3) (2005) 175-191.

[40] R. Andersen, et al., Treatment of periodontal disease by photodisinfection compared to scaling and root planing, J. Clin. Dent. 18 (2) (2007) 34-38.

[41] M. Berakdar, et al., Comparison between scaling-root-planing (SRP) and SRP/ photodynamic therapy: six-month study, Head Face Med 8 (2012) 12.

[42] J. Betsy, et al., Efficacy of antimicrobial photodynamic therapy in the management of chronic periodontitis: a randomized controlled clinical trial, J. Clin. Periodontol. 41 (6) (2014) 573-581.

[43] A. Braun, et al., Short-term clinical effects of adjunctive antimicrobial photodynamic therapy in periodontal treatment: a randomized clinical trial, J. Clin. Periodontol. 35 (10) (2008) 877-884.

[44] N. Christodoulides, et al., Photodynamic therapy as an adjunct to non-surgical periodontal treatment: a randomized, controlled clinical trial, J. Periodontol. 79 (9) (2008) 1638-1644.

[45] L. Ge, et al., Adjunctive effect of photodynamic therapy to scaling and root planing in the treatment of chronic periodontitis, Photomed. Laser Surg. 29 (1) (2011) 33-37.

[46] M. Petelin, et al., Effect of repeated adjunctive antimicrobial photodynamic therapy on subgingival periodontal pathogens in the treatment of chronic periodontitis, Lasers Med. Sci. 30 (6) (2015) 1647-1656.

[47] L.H. Theodoro, et al., Clinical and microbiological effects of photodynamic therapy associated with nonsurgical periodontal treatment. A 6-month follow-up, Lasers Med. Sci. 27 (4) (2012) 687-693.

[48] B.W. Sigusch, et al., Full-mouth antimicrobial photodynamic therapy in Fusobacterium nucleatum-infected periodontitis patients, J. Periodontol. 81 (7) (2010) 975-981.

[49] P. Chondros, et al., Photodynamic therapy as adjunct to non-surgical periodontal treatment in patients on periodontal maintenance: a randomized controlled clinical trial, Lasers Med. Sci. 24 (5) (2009) 681-688.

[50] R. Polansky, et al., Clinical effectiveness of photodynamic therapy in the treatment of periodontitis, J. Clin. Periodontol. 36 (7) (2009) 575-580.

[51] S.L. Pinheiro, et al., Capacity of photodynamic therapy for microbial reduction in periodontal pockets, Lasers Med. Sci. 25 (1) (2010) 87-91.

[52] R. Pourabbas, et al., Effects of photodynamic therapy on clinical and gingival crevicular fluid inflammatory biomarkers in chronic periodontitis: a split-mouth randomized clinical trial, J. Periodontol. 85 (9) (2014) 1222-1229.

[53] N.P. Lang, M.S. Tonetti, Periodontal risk assessment (PRA) for patients in suppor tive periodontal therapy (SPT), Oral Health Prev. Dent. 1 (1) (2003) 7-16.

[54] M.A. Atieh, Photodynamic therapy as an adjunctive treatment for chronic periodontitis: a meta-analysis, Lasers Med. Sci. 25 (4) (2010) 605-613.

[55] J.A. Keestra, et al., Non-surgical periodontal therapy with systemic antibiotics in patients with untreated chronic periodontitis: a systematic review and meta-analysis, J. Periodontal Res. 50 (3) (2015) 294-314.

[56] T.F. Flemmig, et al., Differential clinical treatment outcome after systemic metronidazole and amoxicillin in patients harboring Actinobacillus actinomycetemcomitans and/or Porphyromonas gingivalis, J. Clin. Periodontol. 25 (5) (1998) 380-387.

[57] N.J. Lopez, J.A. Gamonal, B. Martinez, Repeated metronidazole and amoxicillin treatment of periodontitis. A follow-up study, J. Periodontol. 71 (1) (2000) 79-89.

[58] A. Mombelli, et al., Enamel matrix proteins and systemic antibiotics as adjuncts to non-surgical periodontal treatment: clinical effects, J. Clin. Periodontol. 32 (3) (2005) 225-230.

[59] B. Ehmke, et al., Adjunctive antimicrobial therapy of periodontitis: long-term effects on disease progression and oral colonization, J. Periodontol. 76 (5) (2005)
$749-759$.

[60] C. Giannopoulou, et al., Enamel matrix derivative and systemic antibiotics as adjuncts to non-surgical periodontal treatment: biologic response, J. Periodontol. 77 (4) (2006) 707-713.

[61] R. Cosgarea, et al., Non-surgical periodontal treatment in conjunction with 3 or 7 days systemic administration of amoxicillin and metronidazole in severe chronic periodontitis patients. A placebo-controlled randomized clinical study, J. Clin. Periodontol. 43 (9) (2016) 767-777.

[62] C. Giannopoulou, et al., Systemic biomarkers in 2-phase antibiotic periodontal treatment: a randomized clinical trial, J. Dent. Res. 95 (3) (2016) 349-355.

[63] H.F. Jentsch, et al., Nonsurgical therapy of chronic periodontitis with adjunctive systemic azithromycin or amoxicillin/metronidazole, Clin Oral Investig 20 (7) (2016) 1765-1773.

[64] A. Saleh, et al., Comparison of adjunctive azithromycin and amoxicillin/metronidazole for patients with chronic periodontitis: preliminary randomized control trial, Aust. Dent. J. 61 (4) (2016) 469-481.

[65] T. Berglundh, et al., The use of metronidazole and amoxicillin in the treatment of advanced periodontal disease. A prospective, controlled clinical trial, J. Clin. Periodontol. 25 (5) (1998) 354-362.

[66] T.S. Miranda, et al., Metronidazole and amoxicillin as adjuncts to scaling and root planing for the treatment of type 2 diabetic subjects with periodontitis: 1 -year outcomes of a randomized placebo-controlled clinical trial, J. Clin. Periodontol. 41 (9) (2014) 890-899.

[67] G.M. Soares, et al., Metronidazole alone or with amoxicillin as adjuncts to nonsurgical treatment of chronic periodontitis: a secondary analysis of microbiological results from a randomized clinical trial, J. Clin. Periodontol. 41 (4) (2014) 366-376.

[68] A. Guerrero, et al., Adjunctive benefits of systemic amoxicillin and metronidazole in non-surgical treatment of generalized aggressive periodontitis: a randomized placebo-controlled clinical trial, J. Clin. Periodontol. 32 (10) (2005) 1096-1107.

[69] A. Guerrero, J.J. Echeverria, M.S. Tonetti, Incomplete adherence to an adjunctive systemic antibiotic regimen decreases clinical outcomes in generalized aggressive periodontitis patients: a pilot retrospective study, J. Clin. Periodontol. 34 (10) (2007) 897-902.

[70] S.S. Oberoi, et al., Antibiotics in dental practice: how justified are we, Int. Dent. J. 65 (1) (2015) 4-10.

[71] M. Wainwright, Photodynamic antimicrobial chemotherapy (PACT), J. Antimicrob Chemother. 42 (1) (1998) 13-28.

[72] M. Lulic, et al., One-year outcomes of repeated adjunctive photodynamic therapy during periodontal maintenance: a proof-of-principle randomized-controlled clinical trial, J. Clin. Periodontol. 36 (8) (2009) 661-666.

[73] V.S. Muller Campanile, et al., Single or repeated antimicrobial photodynamic therapy as adjunct to ultrasonic debridement in residual periodontal pockets: clinical, microbiological, and local biological effects, Lasers Med. Sci. 30 (1) (2015) 27-34.

[74] G.B. Anderson, et al., Effectiveness of subgingival scaling and root planing: single versus multiple episodes of instrumentation, J. Periodontol. 67 (4) (1996) 367-373.

[75] W.M. Jenkins, et al., Effect of subgingival scaling during supportive therapy, J. Clin. Periodontol. 27 (8) (2000) 590-596.

[76] M.F. Kolbe, et al., Photodynamic therapy during supportive periodontal care: clinical, microbiologic, immunoinflammatory, and patient-centered performance in a split-mouth randomized clinical trial, J. Periodontol. 85 (8) (2014) e277-86.

[77] N.B. Arweiler, et al., Six-month results following treatment of aggressive periodontitis with antimicrobial photodynamic therapy or amoxicillin and metronidazole, Clin Oral Investig 18 (9) (2014) 2129-2135.

[78] V.G. Garcia, et al., Adjunctive antimicrobial photodynamic treatment of experimentally induced periodontitis in rats with ovariectomy, J. Periodontol. 84 (4) (2013) 556-565.

[79] C.J. Smiley, et al., Systematic review and meta-analysis on the nonsurgical treatment of chronic periodontitis by means of scaling and root planing with or without adjuncts, J. Am. Dent. Assoc. 146 (7) (2015) 508-524 (e5). 